\title{
Correction to "RNA Editing Enzymes Modulate the Expression of Hepatic CYP2B6, CYP2C8, and Other Cytochrome P450 Isoforms"
}

\author{
In the above article [Nozaki K, Nakano M, Iwakami C, Fukami T, and Nakajima M (2019) Drug Metab \\ Dispos 47(6): 639-647; DOI: https://doi.org/10.1124/dmd.119.086702], several errors were introduced \\ during copyediting.
}

First, an incorrect version of Figure 6 was published. In panel $H$, the "b" should be a " $\beta$ ". The corrected Figure 6 is displayed below. 
A

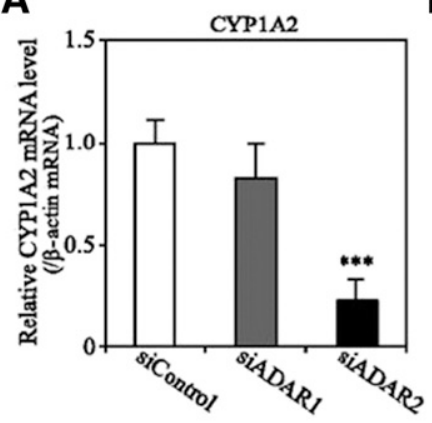

D

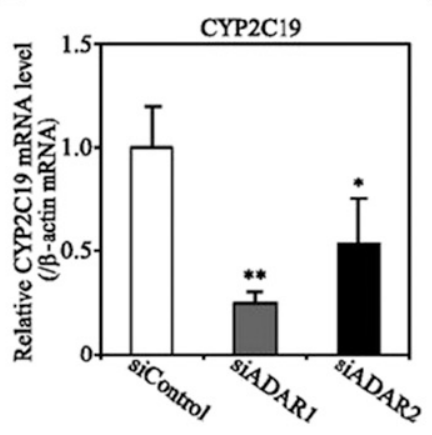

B

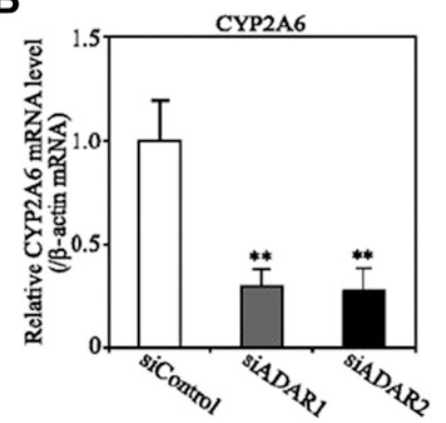

$\mathbf{E}$

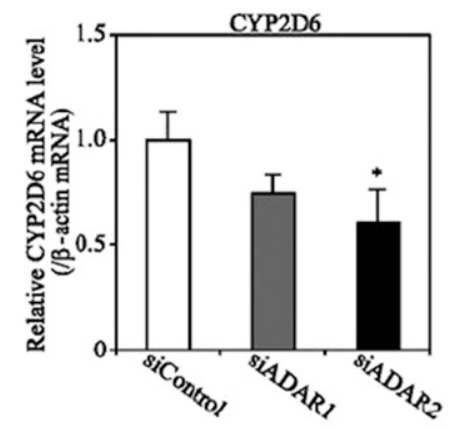

C

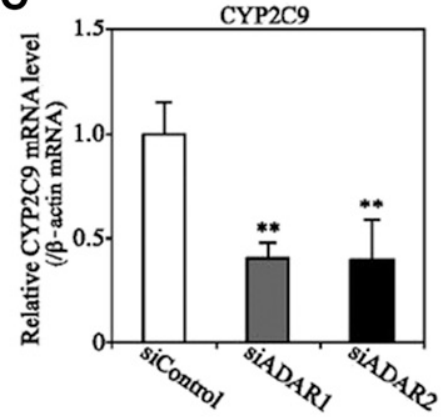

$\mathbf{F}$

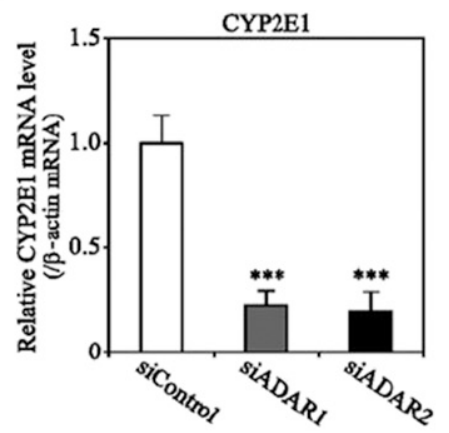

G

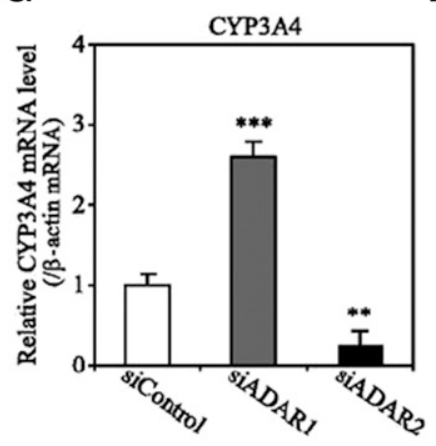

I

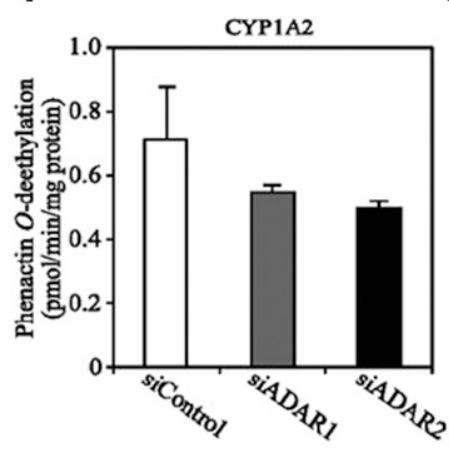

H

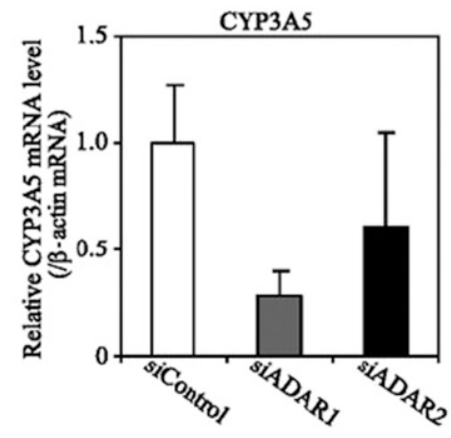

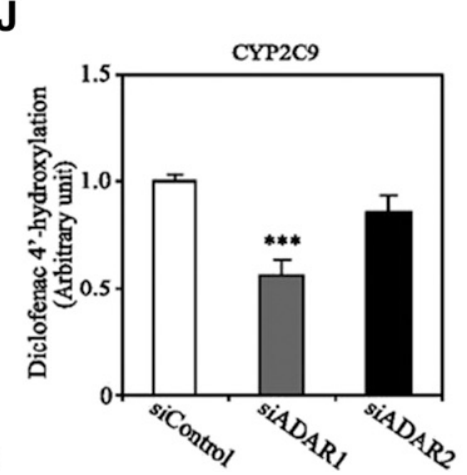

$\mathbf{K}$

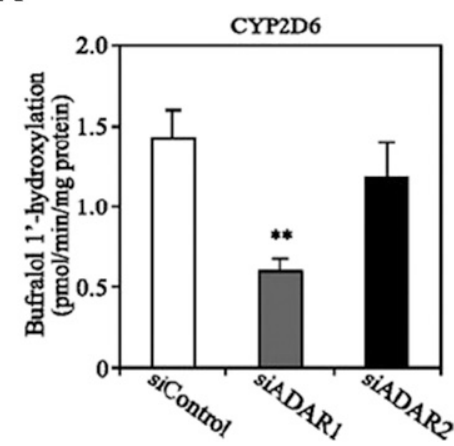

The second error appeared in Table 2 where hyphens were incorrectly set in the terms $N$-Desethylamodiaquine, 4'-Hydroxydiclofenac, and 1'-Hydroxybufuralol. The corrected Table 2 is displayed below. 
TABLE 2

Conditions of liquid chromatography-tandem mass spectrometry

\begin{tabular}{lllll}
\hline \multirow{2}{*}{ Isoform } & \multirow{2}{*}{ Metabolite } & \multicolumn{2}{c}{$\mathrm{m} / \mathrm{z}$} & \multirow{2}{*}{ Collision Energy } \\
\cline { 3 - 4 } & & Precursor Ion & Production & \\
\hline & & & $\mathrm{eV}$ \\
CYP1A2 & Acetaminophen & 152.05 & 110.00 & -16 \\
CYP2C8 & $N$-Desethylamodiaquine & 328.10 & 283.05 & -19 \\
CYP2C9 & $4^{\prime}$-Hydroxydiclofenac & 311.15 & 267.10 & 10 \\
CYP2D6 & 1'-Hydroxybufuralol & 278.35 & 186.05 & -18 \\
\hline
\end{tabular}

$\mathrm{m} / \mathrm{z}$, mass-to-charge ratio.

The third error appeared on page 640, in the Materials and Methods section. In the third sentence, a hyphen was incorrectly added to the term 1'-Hydroxybufuralol. The correct term is1'-Hydroxybufuralol.

The HTML and PDF versions of the article have been corrected.

The compositor apologizes for the errors and any inconvenience they caused. 